\title{
Molecular Characterization of Fusarium Head Blight Pathogens Sampled from a Naturally Infected Disease Nursery Used for Wheat Breeding Programs in China
}

K. D. Puri, Department of Plant Pathology, North Dakota State University, Fargo 58108; E. S. Saucedo, Department of Microbiology, Northern Arizona University, Flagstaff 86011; and S. Zhong, Department of Plant Pathology, North Dakota State University

\begin{abstract}
Puri, K. D., Saucedo, E. S., and Zhong, S. 2012. Molecular characterization of Fusarium head blight pathogens sampled from a naturally infected disease nursery used for wheat breeding programs in China. Plant Dis. 96:1280-1285.

Fusarium head blight (FHB) is an important disease of wheat and barley worldwide. The disease is primarily caused by members of the Fusarium graminearum species complex, consisting of at least 14 phylogenetically distinct species. To determine the population structure of the FHB pathogens in a naturally infected disease nursery located at Jianyang, Fujian province, China, 160 isolates of the $F$. graminearum complex were recovered from symptomatic wheat spike samples collected in two consecutive years (2008 and 2009) and characterized using species- and chemotype-specific polymerase chain reaction as well as variable number tandem repeat (VNTR) markers. All isolates analyzed were identified as $F$. asiaticum except for one isolate, which was identified as $F$. avenaceum. Among the $159 \mathrm{~F}$. asiaticum isolates, $126(79 \%)$ isolates were of the nivalenol (NIV) type while $29(18 \%)$ isolates were of the 15-acetyl deoxynivalenol type and only $4(3 \%)$

isolates were of the 3-acetyl deoxynivalenol type. The 10 VNTR markers revealed 124 distinct haplotypes and 76 polymorphic alleles across the whole population. The two subpopulations (FA-08 and FA-09) grouped based on the year of collection exhibited low genetic differentiation $\left(F_{s t}=0.032\right)$ and high gene flow $\left(N_{m}=15.13\right)$. However, a significant genetic differentiation was found within the NIV-type isolates as revealed by the Structure software. The pairwise linkage disequilibrium tests did not support the hypothesis of random mating in the population because half $(48.8 \%)$ of the locus pairs showed a linkage disequilibrium $(P>0.05)$. Our results suggest that FHB in this nursery was caused by a genetically homogenous and non-random mating population of $F$. asiaticum in 2008 and 2009, which consisted of all three trichothecene types with various levels of aggressiveness.
\end{abstract}

Fusarium head blight (FHB), or scab, is a major disease of wheat and barley worldwide. The disease causes significant losses in yield and quality of the two crops $(23,46)$. In North America, more than $\$ 3$ billion of losses were estimated after the FHB epidemics during the 1990s (23). Frequent disease outbreaks also have been reported from Asia, Europe, and South America in recent years, posing a potential threat to the world's grain production and food supply (17). In China, the first FHB outbreak occurred in 1936 in Anhui province but epidemics were sporadic for almost a half century after that year (47). Since 1985, the disease has become more frequent and widespread in major wheat-growing areas of eastern and central China, especially along the lower and middle reaches of the Yangtze River Valley as well as the northeastern and central parts of China $(3,35)$.

Several Fusarium spp. can cause FHB but the predominant pathogens vary with regions and may change over time. In many regions of the world, FHB is mainly caused by members of the Fusarium graminearum species complex, consisting of at least 14 phylogenic species $(28,29,38,50)$. In China, F. graminearum sensu lato was found in $94.5 \%$ of the samples recovered from 20 provinces along the Yangtze River Valley and 17 other Fusarium spp. were also isolated (43). Global studies of Fusarium spp. composition and population structure showed that $F$. graminearum sensu stricto, one member of the $F$. graminearum complex, is dominant in North America $(28,29,38)$, although other species were also found in some other regions of the United States (15). In China, $F$. asiaticum and $F$. graminearum sensu stricto are the dominant species to initiate FHB in wheat $(14,35,54)$ and barley $(48,53,55)$, with $F$. asiaticum being more common in the southern China and $F$.

Corresponding author: S. Zhong, E-mail: shaobin.zhong@ndsu.edu

Accepted for publication 6 April 2012.

http://dx.doi.org/10.1094/PDIS-08-11-0713-RE

(C) 2012 The American Phytopathological Society graminearum sensu stricto being more frequent in the north (35). $F$. asiaticum and $F$. graminearum were also found to be the predominant etiological agents of FHB in Japan, although their distributions vary depending on the regions $(19,20,40)$.

Population subdivision of $F$. graminearum correlates to geographical differences and trichothecene types worldwide $(14,16,45,48)$. Previously, Karugia et al. (19) found $42.3 \%$ of isolates were of nivalenol (NIV) type and $57.7 \%$ were of 3-acetyl deoxynivalenol (3ADON) type but no 15 -acetyl deoxynivalenol (15ADON)-type isolates were identified among 208 isolates collected from Zhejiang province in China. However, a recent study of $448 \mathrm{~F}$. asiaticum isolates collected from barley at 18 sampling sites along the Yangtze River Valley identified all three trichothecene types $(\mathrm{NIV}=109,3 \mathrm{ADON}=159$, and $15 \mathrm{ADON}=14)(53)$. They also found significant genetic differentiation among populations originated from upper, middle, and lower regions of Yangtze River. Earlier studies on population genetics of $F$. graminearum indicated a relatively homogenous population in the United States $(51,52)$, but more recent studies revealed genetically divergent populations in the Northern Great Plains of the United States and Canada $(16,34,45)$.

Although population genetics of FHB pathogens have been studied extensively on a global to regional scale $(16,35,45,48,52$, 53,55), information on the species composition, trichothecene types, and genetic structure of the pathogen population from a smaller area or experimental unit is relatively limited. In Jianyang, Fujian province of China, where the weather is consistently warm and humid during and after wheat anthesis, FHB develops very well under natural infection conditions every year $(3,56)$. For this reason, a scab nursery was established in the early 1970s and has been used for screening breeding lines or other materials of wheat for FHB resistance ever since. However, the pathogen profile of the nursery is not known. Studies of the genetic structure of pathogen populations not only may provide important information regarding the epidemiology and evolutionary potential of the $F$. graminearum complex but also could lead to improved strategies for controlling this pathogen (51). We attempted to answer several questions, in- 
cluding (i) how many species of the $F$. graminearum complex caused FHB in the nursery, (ii) what were the number and relative frequency of trichothecene types, and (iii) was there genetic differentiation in the pathogen population? Therefore, the major objectives of our study were to determine (i) species composition, (ii) trichothecene types, and (iii) genetic structure of FHB pathogens from this small (approximately $500 \mathrm{~m}^{2}$ ) disease nursery under natural infection.

\section{Materials and Methods}

Fungal isolates. Symptomatic wheat heads were sampled from an FHB nursery (10 by $50 \mathrm{~m}^{2}$ in size) located at Jianyang, Fujian province, China (Fig. 1), in two consecutive years (2008 and 2009). In 2008, wheat spikes showing FHB symptoms were randomly collected from representative plots of the nursery. In 2009, two to three scabby heads were sampled from each of 54 spots evenly distributed in the nursery, with a distance of 5 to $10 \mathrm{~m}$ between two neighboring spots. Fungal isolation, growth, and storage were done as described by Puri and Zhong (34). Each isolate was named after JY (Jianyang, the nursery location) followed by 08 or 09 (collection year) and serial numbers. For example, JY08-001 represents the isolate collected from Jianyang in 2008 with a serial number 001.

DNA extraction, Fusarium spp. identification, and trichothecene type determination. For DNA isolation, mycelia were har-

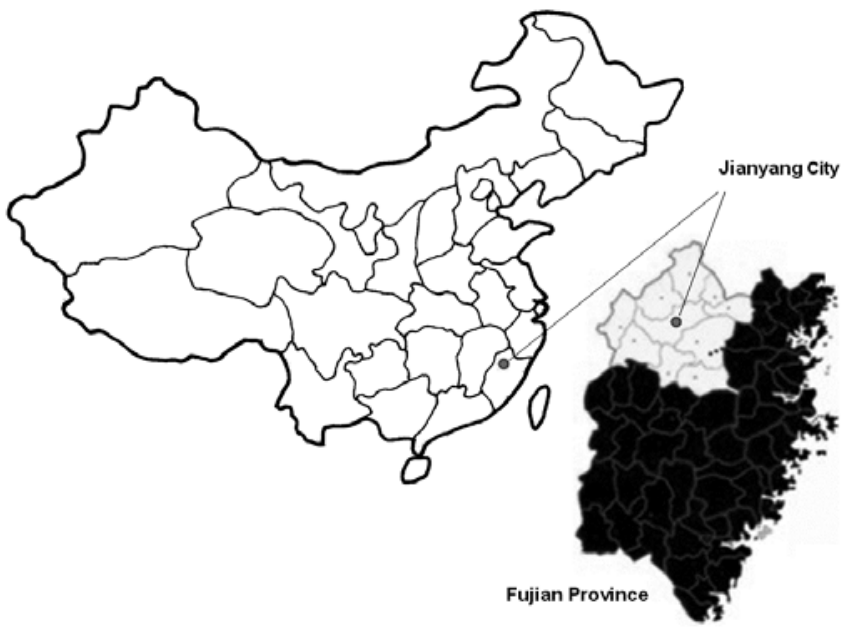

Fig. 1. Map of Fujian province (lower right), which is located in the southeast region of China (upper). The naturally infected Fusarium head blight (FHB) disease nursery is at the city of Jianyang, north of Fujian province. Symptomatic wheat heads were randomly collected from a field $\left(10\right.$ by $\left.50 \mathrm{~m}^{2}\right)$ in the nursery for two consecutive years (2008 and 2009). vested from a single spore-derived culture of each fungal isolate grown on cellophane layered over half-strength potato dextrose agar for 6 to 7 days. DNA was extracted using the FastDNA Kit and FastPrep instrument (MP Biomedical) according to the manufacturer's instructions, and then quantified using a NanoDrop (NanoDrop Technologies). To confirm the identity of the Fusarium spp., a portion of the histone $H 3$ gene was amplified using polymerase chain reaction (PCR) according to the method described by O'Donnell et al. $(28,29)$. The amplicons were purified by ExoSAP IT (USB Corporation) and sent to the University of Hawaii for sequencing. The isolates with identical DNA sequences were grouped together. The sequences (JQ435850 to JQ435858) from the representative isolates in each of the groups were used for BLASTN search against the National Center for Biotechnology Information GenBank database. The primers (6A3AF, 6A3AR, 6CNF, 6ANR, 6G3AF, 6G3AR, 3D15AF, and 3D15AR) developed and described by Suzuki et al. (41) were used to differentiate $F$. graminearum and $F$. asiaticum. Trichothecene types were determined by PCR using the trichothecene-specific multiplex primers and conditions previously described $(38,44)$. The PCR products were separated on a $1 \%$ agarose gel and sized with reference to a 100-bp DNA ladder (NEB). The amplicon at 840, 610, and 243 bp corresponds to the NIV, 15ADON, or 3ADON type, respectively (44).

Analysis of variable number tandem repeats. Nine variable number tandem repeat (VNTR) markers (HK913, HK917, HK957, HK965, HK967, HK977, HK1043, HK1059, and HK1073) developed by Suga et al. (39) and one additional VNTR marker HK1003, described by Gale et al. (13) were used to analyze the population structure of the FHB pathogens. Information of the primer pairs used to detect the 10 VNTR markers is provided in Table 1 . The forward primer of each primer pair was labeled with IRD700/800 at the $5^{\prime}$ end (Eurofins MWG Operon). PCR was performed as described by Suga et al. (39), with some modifications. Each PCR reaction was run in a total volume of $10 \mu$ l containing $1 \times$ reaction buffer, $200 \mu \mathrm{M}$ dNTP, $1 \mu \mathrm{M}$ each primer (forward and reverse), 1 unit of Taq polymerase, and genomic DNA at 25 to $30 \mathrm{ng} / \mu \mathrm{l}$ on a PTC-100 thermal cycler (MJ Research). Amplification profile consisted of initial denaturation at $94^{\circ} \mathrm{C}$ for $3 \mathrm{~min}$; followed by 25 cycles of $94^{\circ} \mathrm{C}$ for $30 \mathrm{~s}, 52^{\circ} \mathrm{C}$ for $30 \mathrm{~s}$, and $72^{\circ} \mathrm{C}$ for $45 \mathrm{~s}$; and a final extension at $72^{\circ} \mathrm{C}$ for $5 \mathrm{~min}$. The PCR products were detected on the Li-COR system (Li-COR) according to the method previously described by Puri and Zhong (34). For each primer pair, amplicons with the same size were considered as the same allele and scored on size (bp). A binary system (i.e., $1=$ present and $0=$ absent) was also used as required for analysis.

Population genetic analysis. Initially, we used Structure 2.3.2 software based on a Bayesian-model-based clustering method $(12,33)$ to assign each multilocus genotype to user-defined genetic $K$ clusters. The analysis was performed with $K=1$ to 5 for five

Table 1. Number of alleles, gene diversity, and fixation index $\left(G_{s t}\right)$ of 10 variable number tandem repeat (VNTR) markers used to analyze the 144 Fusarium asiaticum isolates collected from the disease nursery an Jianyang, China

\begin{tabular}{llccccc}
\hline & & & \multicolumn{3}{c}{ Gene diversity $^{\mathbf{a}}$} \\
\cline { 4 - 5 } VNTR $^{\mathbf{b}}$ & \multicolumn{1}{c}{ Location $^{\mathbf{c}}$} & $\boldsymbol{N}^{\mathbf{d}}$ & $\mathbf{2 0 0 8}$ & $\mathbf{2 0 0 9}$ & Overall $^{\boldsymbol{G}_{\boldsymbol{s t}} \mathbf{e}^{\mathbf{e}}}$ \\
\hline HK1043 & Ch1/Ct1.52/41,839 & 3 & $0.38(0.39)$ & $0.44(0.44)$ & $0.42(0.42)$ & 0.002 \\
HK957 & Ch1/Ct1.91/16,055 & 7 & $0.81(0.84)$ & $0.80(0.81)$ & $0.81(0.82)$ & 0.012 \\
HK967 & Ch2/Ct1.154/53,868 & 2 & $0.00(0.00)$ & $0.02(0.02)$ & $0.02(0.02)$ & 0.005 \\
HK1003 & Ch1/Ct4.37/6,986 & 26 & $0.90(0.93)$ & $0.94(0.95)$ & $0.94(0.95)$ & 0.017 \\
HK1073 & Ch4/Ct1.398/70,812 & 12 & $0.75(0.77)$ & $0.78(0.79)$ & $0.78(0.79)$ & 0.022 \\
HK1059 & Ch3/Ct1.196/164,228 & 6 & $0.74(0.76)$ & $0.77(0.78)$ & $0.78(0.78)$ & 0.024 \\
HK977 & Ch3/Ct1.208/47,696 & 4 & $0.10(0.1)$ & $0.29(0.29)$ & $0.24(0.24)$ & 0.020 \\
HK917 & Ch1/Ct1.82/2,471 & 3 & $0.15(0.15)$ & $0.12(0.12)$ & $0.13(0.13)$ & 0.000 \\
HK913 & Ch1/Ct1.73/664 & 6 & $0.74(0.76)$ & $0.63(0.63)$ & $0.70(0.70)$ & 0.064 \\
HK965 & Ch2/Ct1.154/51,671 & 7 & $0.64(0.66)$ & $0.75(0.75)$ & $0.74(0.75)$ & 0.038 \\
Mean & $\ldots$ & 7.6 & $0.52(0.53)$ & $0.55(0.55)$ & $0.56(0.56)$ & 0.026 \\
\hline
\end{tabular}

a Nei's gene diversity $(H)(26)$ within populations was calculated using POPGENE 1.32 (49). Values in parenthesis were calculated by ARLEQUIN 3.1 (10).

${ }^{\mathrm{b}}$ HK1003 was described by Gale et al. (13) and the others were reported by Suga et al. (39).

${ }^{\mathrm{c}}$ Marker location in the genome was indicated by chromosome $(\mathrm{Ch})$ number/contig $(\mathrm{Ct})$ number/position in contig.

${ }^{\mathrm{d}}$ Number of alleles.

${ }^{\text {e }} G_{s t}$ fixation indices (26) for individual loci were calculated for the 132 clone corrected isolates using POPGENE 1.32 (49). 
iterations with 100,000 Markov Chain Monte Carlo reps after burn-in period of 10,000 (33). The exact number of clusters $(K)$ was determined based on ln $(\mathrm{P})$ curve for each subpopulation probability and verified with Structure Harvester, a web-based program to visualize Structure output implementing the Evanno method (8). To test whether there was a genetic differentiation between isolates collected from the 2 years, we also grouped isolates into populations according to the year of collection. Isolates collected from 2008 were grouped into one population (FA-08) and those from 2009 were grouped into another population (FA-09).

The genotypic diversity $(G D)$ and index of multilocus linkage disequilibrium $\left(\bar{r}_{d}\right)$ were calculated using software MULTILOCUS 1.3 with the algorithm $G=(n / n-1)\left\{\left(1-\Sigma p_{\mathrm{i}}^{2}\right)\right\}$, where $n$ is the number of individuals sampled and $p_{\mathrm{i}}$ is the frequency of $i$ th genotype (1). The $G D$ has a value of 0 if every individual is of the same genotype or 1 if every individual is different in the population. The index $\left(\bar{r}_{d}\right)$ was used to test a hypothesis of random mating within a subpopulation after 1,000 permutations. The index $\left(\bar{r}_{d}\right)$ gives a value of zero if there is no association among alleles on unlinked loci, as expected on null hypothesis of random mating. The significant linkage disequilibrium (LD) among VNTR loci and successive $P$ values were generated using a Marcov chain length of $10^{4}$ and dememorization of $10^{4}$, as implemented in ARLEQUIN 3.1.

The genetic differentiation and other population parameters were determined using different statistical tools and techniques. Analysis of molecular variance (AMOVA), population differentiation or fixation index $\left(F_{s t}\right)$, and number of migrants exchanged between two populations or gene flow $\left(N_{m}\right)$ were calculated using ARLEQUIN 3.11 (10). The allele frequencies, percentage polymorphic loci, gene diversity $(26,27)$, Nei's unbiased genetic distance $(D)$ (27), and fixation index $\left(G_{s t}\right)$ for individual loci were calculated using POPGENE ver. 1.32 (49). Molecular variance (AMOVA) was performed to test hierarchical partition of genetic variation between two populations and individuals within the whole population (11). The gene flow was calculated by $\left.N_{m}=\left(1-F_{s t}\right) / 2 F_{s t}\right)$, where $N$ is the effective population size and $m$ is the migration rate per generation. The statistical significance of pairwise $F_{s t}$ was tested by 1,000 permutations.

Aggressiveness evaluation of representative $F$. asiaticum isolates. A greenhouse experiment was conducted to test the aggressiveness of 16 representative isolates of $F$. asiaticum, including 6 15ADON-type isolates, 4 3ADON-type isolates, and 6 NIV-type isolates, on the spring wheat 'Briggs' (susceptible to FHB). Seed were sown in plastic buckets ( 30 by 20 by $35 \mathrm{~cm}^{3}$ ) filled with Sunshine pot mix (Sun Gro Horticulture Canada Ltd.) with two rows ( 8 seeds/row) per bucket. For each isolate, three rows (replicates) of the plants were used for inoculation at the anthesis. Plant growth conditions, inoculum preparation, inoculation, and disease scoring were the same as previously described (34), except that the inoculated plants were kept in a humidity chamber for $72 \mathrm{~h}$ instead of overhead misting.

\section{Results}

Fusarium spp. identification. In total, 160 Fusarium isolates (59 from 2008 and 101 from 2009) were first analyzed using the
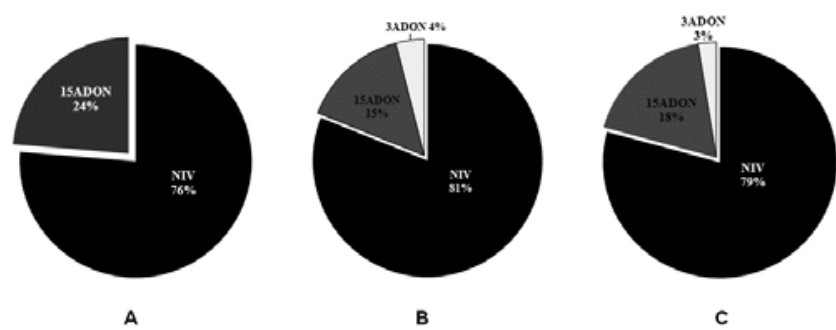

Fig. 2. Frequency distribution of chemotypes among Fusarium asiaticum isolates collected in A, 2008 (59 isolates); B, 2009 (100 isolates); and C, the 2 years combined (159 isolates). 15ADON = 15-acetyl deoxynivalenol, NIV = nivalenol, and $3 A D O N=3$-acetyl deoxynivalenol. species-specific multiplex PCR (41). The result indicated that all isolates except isolate JY09-030 showed the amplicons unique to $F$. asiaticum. Further analysis of histone $H 3$ gene sequences indicated that JY09-030 (JQ435857) was F. avenaceum and the rest of isolates were identified as $F$. asiaticum. Compared with the histone $H 3$ gene sequence of the $F$. asiaticum strain ( $F$. graminearum species complex number 9086 ), $92 \%$ of the $F$. asiaticum isolates analyzed in this study had no or one base difference and only $8 \%$ isolates had two to four base differences.

Trichothecene type determination. Trichothecene types were determined for the $159 \mathrm{~F}$. asiaticum isolates using the multiplex PCR $(25,28)$. Among them, $126(79 \%)$ of the isolates were of the NIV type while $29(18 \%)$ and $4(3 \%)$ isolates were of 15 ADON and 3ADON types, respectively (Fig. 2C). In 2008, 45 (76\%) isolates were of the NIV type and $14(24 \%)$ isolates were of the $15 \mathrm{ADON}$ type but no 3ADON type was detected (Fig. 2A). In 2009 , all three trichothecene types were identified, with $81(81 \%)$ being of the NIV type, 15 (15\%) being of the 15ADON type, and 4 (4\%) being of the 3ADON type (Fig. 2B).

Population genetics analyses. In all, $144 F$. asiaticum isolates were analyzed using the 10 VNTR markers, and 76 polymorphic alleles were generated among them. The number of alleles generated by individual VNTR markers varied from 2 to 26 (Table 1). Gene diversity $(H)$ also differed among the 10 VNTR markers, with HK1003 being the highest (0.94), followed by HK957 (0.82), HK1073 (0.78), and HK1059 (0.78). Marker HK967 and HK917 had the lowest $H(0.02$ and 0.13 , respectively; Table 1$)$. Using POPGENE, a fixation index $\left(G_{s t}\right)$ was generated for each VNTR marker. Marker HK917 had the highest $G_{s t}$ value (0.064) followed by marker HK965 (0.038). However, the three markers named HK917 $\left(G_{s t}=0\right)$, HK1043 $\left(G_{s t}=0.002\right)$, and HK967 $\left(G_{s t}=0.005\right)$ had very low fixation indices (Table 1 ).

Of the 144 isolates, 132 isolates remained after clone removal. All clonal isolates were found in 2008 and clones with the same haplotype belonged to the same trichothecene type. Using Structure, we assigned the 131 (excluding $F$. avenaceum) clone-corrected isolates into distinct clusters. Assignment of isolates into a specific genetic cluster was based on the average membership value $q=0.8$ or above. Using this standard, 98 isolates, in total, were assigned into one of the three clusters (numbers 1,2, and 3 ) with the highest probability. However, 33 isolates did not fit to any of the clusters and, thus, were not assigned into any populations. Cluster $1(q$ mean $=0.941$, standard deviation $[\mathrm{SD}]=0.036)$ had a total 45 isolates consisting of 44 NIV-type isolates and 1 15ADON-type isolate $(q=0.978)$, which was named as population NIV-1. Cluster $2(q$ mean $=0.908, \mathrm{SD}=0.053)$ had 18 isolates comprising 16 NIV-type and 2 15ADON-type isolates and named population NIV-2. Because cluster $3(q$ mean $=0.937, \mathrm{SD}=0.042)$ consisted of 18 NIV-type, 15 15ADON-type, and 2 3ADON-type isolates, we further divided them into two subpopulations, one

Table 2. Nei's genetic distance D (above diagonal) and $P$ value from the exact tests for genetic differentiation $Ф P T$ (below diagonal) between four populations of Fusarium asiaticum collected from the Fusarium head blight disease nursery in China ${ }^{\mathrm{a}}$

\begin{tabular}{lcccc}
\hline Populations $^{\text {b }}$ & NIV-1 & NIV-2 & NIV-3 & DON \\
\hline NIV-1 & $\ldots$ & 0.240 & 0.299 & 0.419 \\
NIV-2 & $<0.001$ & $\ldots$ & 0.346 & 0.399 \\
NIV-3 & $<0.001$ & $<0.001$ & $\ldots$ & 0.076 \\
DON & $<0.001$ & $<0.001$ & 0.139 & $\ldots$ \\
\hline
\end{tabular}

${ }^{a} \mathrm{D}(25)$ was calculated using POPGENE (49). ФPT (analogous to $F_{s t}$ ) was calculated as proportion of variance among and within population relative to the total variance (11) using GENALEX 6.4 (31). ФPT = AP/(WP + $\mathrm{AP})=\mathrm{AP} / \mathrm{TOT}$, where $\mathrm{AP}=$ estimated variance among population, $\mathrm{WP}=$ estimated variance with in population, and TOT = total variance. The probability of obtaining equal or lower $\Phi$ value was determined by 1,000 random permutations.

b The four populations-NIV-1, NIV-2, NIV-3, and DON-are genetically defined and subdivided according to the member assignment by STRUCTURE and by taking into account the trichothecene types. 
named NIV-3, containing all NIV isolates, and the other named DON, consisting of the 3ADON-type and 15ADON-type isolates.

AMOVA among the four populations (NIV-1, NIV-2, NIV-3, and DON) indicated that $17 \%$ of genetic variation was due to differences among populations and $83 \%$ of variation was from individuals within population. The three populations NIV-1, NIV-2, and DON had a similar level of gene diversity $(0.41$ to 0.46$)$ whereas NIV-3 had a relatively high gene diversity (0.54) across all loci. Population-wise comparison showed a significant differentiation among them, except between NIV-3 and DON (Table 2). A high gene flow (25.9) and a very low population differentiation (0.019 and $P=0.139$ ) was found between NIV-3 and DON.

The genetic distance was low $(D=0.05)$ and genetic identity was high $(I=0.95)$ between the two populations (FA-08 and FA09) grouped according to the years of collection. The hierarchical AMOVA showed that genetic variation between the populations accounted for only $3.23 \%$ while most of the genetic variation $(96.77 \%)$ was from individuals within population. Genetic differentiation was low $\left(F_{s t}=0.032\right)$ and gene flow was high $\left(N_{m}=\right.$ 15.13) between FA-08 and FA-09.

The $\bar{r}_{d}$ index was low in both populations (i.e., 0.073 for FA-08 and 0.050 for FA-09) but was significantly different from zero at $P$ $<0.001$. The index of association, another measure of linkage disequilibrium, was 0.575 and 0.424 for FA-08 and FA-09, respectively, and was also significantly different from zero $(P<0.001)$. Analysis of linkage disequilibrium between loci pairs of the 10 VNTR loci showed that almost half $(48.8 \%)$ of the 45 possible locus-pair combinations were in linkage disequilibrium $(P>0.05$; Table 3), suggesting that out-crossing was not frequent in the population.

Aggressiveness evaluation. The average disease severity (DS) caused by individual isolates on Briggs ranged from $12.4 \%$ (JY08013 ) to $96.2 \%$ (JY09-45E). Analysis of variance indicated significant differences $(P>0.0001)$ in aggressiveness among the isolates based on the DS caused on Briggs. Isolate JY09-45E (a 3ADONtype isolate) and JY08-043 (a 15ADON-type isolate) induced the highest DS, which was not significantly different from those induced by isolates JY08-009 and JY09-045 (Fig. 3). However, all

Table 3. Significant linkage disequilibrium $(P<0.05)$ between 10 variable numbers of tandem repeat (VNTR) markers in 131 clone-corrected isolates of Fusarium asiaticum

\begin{tabular}{lc}
\hline VNTR marker pair $^{\mathbf{a}}$ & $\boldsymbol{P}$ value \\
\hline HK1043 (1), HK957 (1) & 0.004 \\
HK1043 (1), HK967 (2) & 0.000 \\
HK1043 (1), HK1073 (4) & 0.004 \\
HK1043 (1), HK1059 (3) & 0.025 \\
HK1043 (1), HK913 (1) & 0.0003 \\
HK957 (1), HK1003 (1) & 0.007 \\
HK957 (1), HK1073 (4) & 0.009 \\
HK957 (1), HK1059 (3) & 0.024 \\
HK957 (1), HK913 (1) & 0.003 \\
HK967 (2), HK913 (1) & 0.013 \\
HK1003 (1), HK1073 (4) & 0.000 \\
HK1003 (1), HK1059 (3) & 0.000 \\
HK1003 (1), HK977 (3) & 0.037 \\
HK1003 (1), HK913 (1) & 0.000 \\
HK1073 (4), HK1059 (3) & 0.000 \\
HK1073 (4), HK917 (1) & 0.004 \\
HK1073 (4), HK913 (1) & 0.004 \\
HK1073 (4), HK965 (2) & 0.036 \\
HK1059 (3), HK913 (1) & 0.010 \\
HK1059 (3), HK965 (2) & 0.032 \\
HK977 (3), HK917 (1) & 0.000 \\
HK913 (1), HK965 (2) & 0.048 \\
\hline
\end{tabular}

${ }^{a}$ Pair-wise estimate of linkage disequilibrium, based on Fisher's exact probability test by Slatkin (37), was calculated for 45 possible marker pairs among 10 VNTR loci using Marcov chain length of $10^{4}$ with $10^{4}$ dememorization implemented in ARLEQUIN 3.1 (10). Only significant loci combinations $(P<0.05)$ were shown in the table. Number in parenthesis is the chromosome on which a particular VNTR locus resides.
NIV-type isolates evaluated were significantly less aggressive compared with the 3ADON and 15ADON isolates (Fig. 3).

\section{Discussion}

Our results indicated that $F$. asiaticum is the primary etiological agent prevalent in the FHB disease nursery. This is consistent with previous studies, which showed that $F$. asiaticum is the predominant and primary pathogen of FHB for all major cereals (wheat, barley, and rice) in warmer regions of Asian countries, including China, Korea, and Japan $(21,35,40)$. The majority of Fusarium isolates collected from FHB-infected wheat and barley samples in eastern and central China along the Yangtze River were identified as $F$. asiaticum in several independent studies $(14,19,20,53,55)$. Qu et al. (35) reported that $79 \%$ of the 437 isolates collected from warmer $\left(>15^{\circ} \mathrm{C}\right)$ regions of China were $F$. asiaticum. Karugia et al. (20) identified $179 \mathrm{~F}$. asiaticum isolates among 183 isolates sampled in a small experimental field in Japan. Lee et al. (21) found that all of the 249 isolates collected from rice fields in southern provinces of Korea were F. asiaticum whereas isolates from eastern provinces of Korea were either $F$. asiaticum or $F$. graminearum sensu stricto. They hypothesize that $F$. asiaticum has a host preference and specificity to rice, and perithecium production typically favors rice straws under warmer conditions. The Jianyang nursery is located in the warmer region, and the field was planted with rice before wheat was grown for FHB evaluation (J. Huang, personal communication). Rice was the major cereal crop in Jianyang and no wheat, barley, or corn were grown around the region. Thus, initial infections might have come from inoculum sources originated from rice straws, as found in Korea (21). However, this hypothesis needs further investigation by comparing isolates originated from the nursery with those from the rice fields surrounding the nursery.

We found that a majority (79\%) of the F. asiaticum isolates were of the NIV type although isolates of the 15ADON type (18\%) and the 3 ADON type (3\%) were also identified. To our knowledge, this is the first time that all three trichothecene types were found in the same place in southeastern China. Karugia et al. (19) analyzed 208 isolates of $F$. asiaticum collected during 2000 by Gale et al. (14) from four locations in Zhejiang province (approximately $500 \mathrm{~km}$ north of Jianyang) and showed that NIV and 3ADON isolates accounted for 42.3 and $57.7 \%$, respectively, but no $15 \mathrm{ADON}$ isolates were detected. Zhang et al. (54) analyzed a 1999 collection of 299 isolates from 12 provinces along the middle and lower reaches of Yangtze River and found that $231(77 \%)$ of the isolates were $F$.

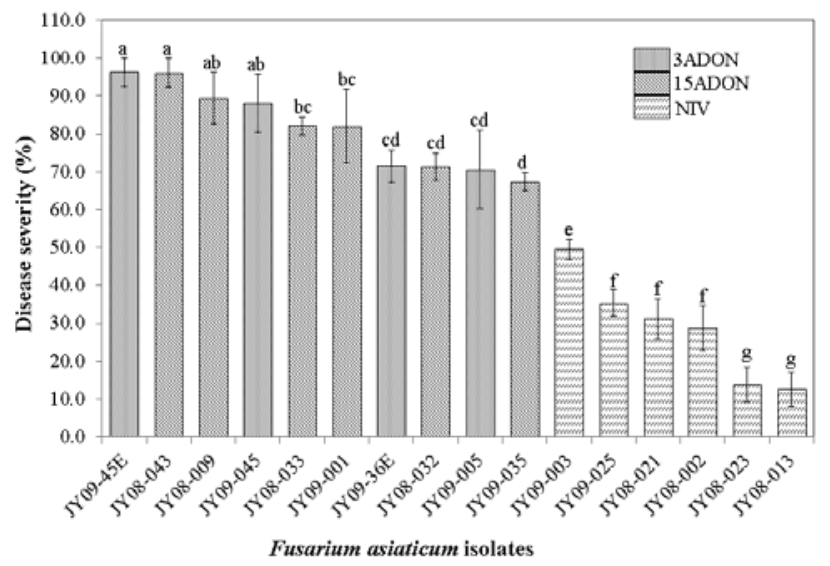

Fig. 3. Average disease severity (DS) caused by each of the 16 representative isolates of Fusarium asiaticum (6 15-acetyl deoxynivalenol [15ADON] isolates, 43 acetyl deoxynivalenol [3ADON] isolates, and 6 nivalenol [NIV] isolates) on the susceptible spring wheat 'Briggs' in the greenhouse. Single-floret inoculation was used and infected spikelets in each of the inoculated spikes were counted at 21 days after inoculation. In general, the 3ADON- and 15ADON-type isolates caused significantly higher DS than the NIV isolates $(P<0.0001)$. Vertical bar represents standard error. Isolates followed by the same letters (indicated at the top of bar) were not significantly different at $P<0.0001$. 
asiaticum. Of the $231 \mathrm{~F}$. asiaticum isolates, $67 \%$ were of the $3 \mathrm{ADON}$ type and $23 \%$ were the NIV type but only $10 \%$ were $15 \mathrm{ADON}$ isolates (54). The higher frequency of $15 \mathrm{ADON}$ isolates found in the present study may reflect a geographical difference between FHB epidemic regions in China because Jianyang is the furthest place in southeast China where $F$. asiaticum isolates have been sampled and analyzed.

Our population genetics study suggested that the two F. asiaticum populations (FA-08 and FA-09) grouped based on the year of collection were genetically similar. This conclusion is supported by the high genetic identity (95\%), high gene flow $\left(N_{m}=15.13\right)$, and very low genetic differentiation $\left(F_{s t}=0.032\right)$ between FA-08 and FA-09. Gale et al. (14) found a high gene flow $\left(N_{m}=7\right.$ to 30$)$ and low $F_{s t}(0.01$ to 0.07$)$ among $F$. asiaticum populations collected from four fields located in Zhejiang province, China. Using the VNTR markers, Karugia et al. (20) analyzed $F$. asiaticum isolates collected in two successive years from a small wheat field $\left(500 \mathrm{~m}^{2}\right)$ in Japan and failed to reveal distinct genetic differentiation between the populations sampled during the 2 years. Zeller et al. (51) also observed a large number of migrants (approximately 70) and high genetic identity (99\%) among $F$. graminearum isolates sampled in two small fields located in North Dakota and Kansas, respectively (51). These results suggest that a pathogen population from a small, isolated disease nursery may keep stable for some period of time. However, population subdivisions based on trichothecene type, geographic difference, and temperature gradient within and outside China from a larger geographic area have been reported by several studies $(16,19,38,44,48,53-55)$. Thus, information from a local population may not be applicable to a larger population across China or vice versa. Population studies on field, regional, and global scales are still necessary to have a better understanding of genetic variation and population structure of the pathogen.

Although little genetic differentiation was found between the two subpopulations (FA-08 and FA-09) grouped based on the years of collection, three distinct subpopulations existed (NIV-1, NIV-2, and NIV-3) within the NIV-type isolates, as revealed by the membership assignment using the Structure software and further confirmed by the other genetic population analyses. Interestingly, the non-NIV-type isolates were clustered with one of the NIV-type subpopulations. This result suggests that a significant genetic differentiation occurred in the pathogen population of the disease nursery. Population subdivision has been reported for wheat and barley isolates of $F$. asiaticum collected along the Yangtze River in southern China (53) and for $F$. graminearum isolates sampled from the United States and Canada $(15,16,34,45)$. The genetic differentiation found between the NIV-type populations (NIV-1, NIV2 , and NIV-3) in our study likely suggests lack of enough sexual recombination between the NIV isolates (see below).

Out-crossing of $F$. graminearum was demonstrated in the laboratory by using nitrate non-utilizing mutants (5). Recently, Chen and Zhou (6) reported out-crossing frequency of 5.7 to $20.9 \%$ in $F$. graminearum in field conditions in China. However, our LD analysis showed that almost half $(48.8 \%)$ of the 45 possible locus pairs were in linkage disequilibrium. This result suggests that out-crossing among isolates of the $F$. asiaticum population in the Jianyang nursery might have been very rare. Previously, Gale et al. (14) found $36 \%$ restriction fragment length polymorphism loci in linkage disequilibrium among the $F$. asiaticum isolates collected from Zhejiang province. Karagua et al. (20) also showed 10 to $12 \%$ of the VNTR loci in linkage disequilibrium condition in Japanese isolates. Thus, out-crossing might not be the major evolutional force for maintaining high genotype diversity of the population in the Jianyang nursery.

Our greenhouse inoculation experiments indicated that the DON-type isolates (15ADON- and 3ADON-type isolates) were similar in aggressiveness whereas they were more aggressive than the NIV-type isolates on Briggs. This result agrees with the previous studies $(15,22)$, which indicated that DON-type isolates are generally more aggressive on wheat compared with the NIV-type isolates. The aggressiveness difference between DON and NIV types may be due to different amounts or types of mycotoxins produced by the FHB pathogens (2). DON-type isolates generally accumulated a higher level of trichothecene toxins than the NIVtype isolates (15; Puri and Zhong et al. unpublished). Previous studies indicated that both DON and NIV are virulence factors, either of which is required for the Fusarium pathogen to cause disease spread in wheat spikes $(4,7,22)$. However, the mechanism of how NIV is involved in pathogen infection and FHB development remains to be investigated.

Among trichothecenes, DON has attracted more attention than NIV (30), which may be due to the fact that DON contaminations were more common in cereal grain samples collected in North America, as is exemplified in the global analysis data of mycotoxin contamination reported by Placinta et al. (32). However, in Asian countries, including China, Japan, Korea, and Vietnam, a high incidence and generally high concentrations of NIV have been reported earlier (42). In a more recent analysis of mycotoxin occurrence and concentrations in food and raw food materials from 12 European countries (36), 16\% of the samples were also found to be positive for NIV. Although several studies suggested that NIV has higher cytotoxicity than DON $(9,24)$ in the experiments with animal or human cells and could be linked to a high incidence of cancer of the esophagus and gastric cardia in China (18), there is limited information on the specific toxic effects of long-term NIV exposure in humans or the comparative toxicity of NIV and DON. Considering the fact that NIV-type Fusarium pathogens are common in Asian countries, Europe, and the southern United States, and NIV-contaminated grains or food products were frequently detected in the FHB-affected samples, more attention should be paid to NIV and its impacts on cereal crop production as well as animal and human consumptions.

In summary, the FHB pathogen population from the small disease nursery was primarily composed of $F$. asiaticum, with the NIV type being predominant. However, isolates of $F$. asiaticum with $3 \mathrm{ADON}$ and $15 \mathrm{ADON}$ types were also present. Population subdivision based on year of collection was not significant, although a genetic differentiation was observed within the NIV-type isolates collected in the 2 years. This study provides important information about the population structure of $F$. graminearum complex in the nursery that has been used for FHB resistance evaluation for more than 25 years. The presence of all trichothecene types, which vary in aggressiveness and mycotoxin production, ensures the value of this particular nursery in FHB resistance evaluation and in breeding wheat cultivars with broad-spectrum FHB resistance.

\section{Acknowledgments}

The work was partially supported by the U.S. Wheat and Barley Scab Initiative. We thank J. Huang for assistance in collecting the FHB samples in the Jianyang nursery.

\section{Literature Cited}

1. Agapow, P. M., and Burt, A. 2001. Indices of multilocus linkage disequilibrium. Mol. Ecol. Notes 1:101-102.

2. Alexander, N. J., McCormick, S. P., Waalwijk, C., van der Lee, T., and Proctor, R. H. 2011. The genetic basis for 3-ADON and 15-ADON trichothecene chemotypes in Fusarium. Fungal Genet. Biol. 48:485-495.

3. Bai, G. H., Chen, L. F., and Shaner, G. E. 2003. Breeding for resistance to head blight of wheat in China. Pages 296-317 in: Fusarium Head Blight of Wheat and Barley. K. J. Leonard and W. R. Bushnell, eds. American Phytopathological Society, St. Paul, MN.

4. Bai, G. H., Desjardins, A. E., and Plattner, R. D. 2001. Deoxynivalenol nonproducing Fusarium graminearum causes initial infection, but does not cause disease spread in wheat spikes. Mycopathologia 153:91-98.

5. Bowden, R. L., and Leslie, J. F. 1999. Sexual recombination in Gibberella zeae. Phytopathology 89:182-188.

6. Chen, Y., and Zhou, M. G. 2009. Sexual recombination of carbendazim resistance in Fusarium graminearum under field conditions. Pest. Manage. Sci. 65:398-403.

7. Desjardins, A. E., Proctor, R. H., Bai, G., McCormick, S. P., Shaner, G., Buechley, G., and Hohn, T. M. 1996. Reduced virulence of trichothecenes antibiotic-nonproducing mutants of Gibberella zeae in wheat field tests. Mol. Plant-Microbe Interact. 9:775-781. 
8. Earl, D. A., and vonHoldt, B. M. 2011. STRUCTURE HARVESTER: a website and program for visualizing STRUCTURE output and implementing the Evanno method. Conservation Genetics Resources. Online publication. doi:10.1007/s12686-011-9548-7

9. Eriksen, G. S., Pettersson, H., and Lundh, T. 2004. Comparative cytotoxicity of deoxynivalenol, nivalenol, their acetylated derivatives and de-epoxy metabolites. Food Chem. Toxicol. 42:619-624.

10. Excoffier, L., Laval, G., and Schneider, S. 2005. Arlequin ver. 3.0: An integrated software package for population genetics data analysis. Evol. Bioinf. Online 1:47-50.

11. Excoffier, L., Smouse, P., and Quattro, J. 1992. Analysis of molecular variance inferred from metric distances among DNA haplotypes, application to human mitochondrial DNA restriction data. Genetics 131:479-491.

12. Falush, D., Stephens, M., and Pritchard, J. K. 2003. Inference of population structure using multilocus genotype data: linked loci and correlated allele frequencies. Genetics 164:1567-1587.

13. Gale, L. R., Bryant, J. D., Calvo, S., Giese, H., Katan, T., O’Donnell, K., Suga, H., Taga, M., Usgaard, T. R., Ward, T. J., and Kistler, H. C. 2005. Chromosome complement of the fungal plant pathogen Fusarium graminearum based on genetic and physical mapping and cytological observations. Genetics 171:985-1001.

14. Gale, L. R., Chen, L. F., Hernick, C. A., Takamura, K., and Kistler, H. C. 2002. Population analysis of Fusarium graminearum from wheat fields in eastern China. Phytopathology 92:1315-1322.

15. Gale, L. R., Harrison, S. A., Ward, T. J., O’Donnell, K., Milus, E. A., Gale, S. W., and Kistler, H. C. 2011. Nivalenol-type populations of Fusarium graminearum and $F$. asiaticum are prevalent on wheat in southern Louisiana. Phytopathology 101:124-134

16. Gale, L. R., Ward, T. J., Balmas, V., and Kistler, H. C. 2007. Population subdivision of Fusarium graminearum sensu stricto in the upper Midwestern United States. Phytopathology 97:1434-1439.

17. Goswami, R. S., and Kistler, H. C. 2004. Heading for disaster: Fusarium graminearum on cereal crops. Mol. Plant Pathol. 5:515-525.

18. Hsia, C. C., Wu, Z. Y., Li, Y. S., Zhang, F., and Sun, Z. T. 2004. Nivalenol, a main Fusarium toxin in dietary foods from high-risk areas of cancer of esophagus and gastric cardia in China, induced benign and malignant tumors in mice. Oncol. Rep. 12:449-456.

19. Karugia, G. W, Suga, H., Gale. L. R., Nakajima, T., Ueda, A., and Hyakumachi, M. 2009. Population structure of Fusarium asiaticum from two Japanese regions and eastern China. J. Gen. Plant Pathol. 75:110-118.

20. Karugia, G. W., Suga, H., Gale, L. R., Nakajima, T., Tomimura, K., and Hyakumachi, M. 2009. Population structure of the Fusarium graminearum species complex from a single Japanese wheat field sampled in two consecutive years. Plant Dis. 93:170-174.

21. Lee, J., Chang, I. Y., Kim, H., Yun, S. H., Leslie, J. F., and Lee, Y. W. 2009. Genetic diversity and fitness of Fusarium graminearum populations from rice in Korea. Appl. Environ. Microbiol. 75:3289-3295

22. Maier, F. J., Miedaner, T., Hadeler, B., Felk, A., Salomon, S., Lemmens, M., Kassner, H., and Schäfer, W. 2006. Involvement of trichothecenes in fusarioses of wheat, barley, and maize evaluated by gene disruption of the trichodiene synthase (tri5) gene in three field isolates of different chemotype and virulence. Mol. Plant Pathol. 7:449-461.

23. McMullen, M., Jones, R., and Gallenberg, D. 1997. Scab of wheat and barley: a re-emerging disease of devastating impact. Plant Dis. 81:3401348.

24. Minervini, F., Fornelli, F., and Flynn, K. M. 2004. Toxicity and apoptosis induced by the mycotoxins nivalenol, deoxynivalenol and fumonisin B1 in a human erythroleukemia cell line. Toxicol. Vitro 18:21-28.

25. Nei, M. 1972. Genetic distance between populations. Am. Nat. 106:283292.

26. Nei, M. 1973. Analysis of gene diversity in subdivided populations. Proc. Natl. Acad. Sci. USA 70:3321-3323.

27. Nei, M. 1978. Estimation of average heterozygosity and genetic distance from a small number of individuals. Genetics 76:379-390.

28. O'Donnell, K., Kistler, H. C., Tacke, B. K., and Casper, H. H., 2000. Gene genealogies reveal global phylogeographic structure and reproductive isolation among lineages of Fusarium graminearum, the fungus causing wheat scab. Proc. Natl. Acad. Sci. USA 97:7905-7910.

29. O’Donnell, K., Ward, T. J., Geiser, D. M., Kistler, H. C., and Aoki, T. 2004. Genealogical concordance between the mating type locus and seven other nuclear genes supports formal recognition of nine phylogenically distinct species within the Fusarium graminearum clade. Fungal Genet. Biol. 41:600-623.

30. Pasquali, M., Giraud, F., Brochot, C., Cocco, E., Hoffman, L., and Bohn, T. 2009. Genetic Fusarium chemotyping as a useful tool for predicting nivalenol contamination in winter wheat. Int. J. Food Microbiol. 137:246253.

31. Peakall, R., and Smouse, P. E. 2006. GENALEX 6: genetic analysis in Excel. Population genetic software for teaching and research. Mol. Ecol. Notes 6:288-295.

32. Placinta, C. M., D'Mello, J. P. F., and MacDonald, A. M. C. 1999. A review of worldwide contamination of cereal grains and animal feed with Fusarium mycotoxins. Anim. Feed Sci. Technol. 78:21-37.
33. Pritchard, J. K., Stephens, M., and Donnelly, P. 2000. Inference of population structure using multilocus genotype data. Genetics 155:945-959.

34. Puri, K. D., and Zhong, S. 2010. The 3ADON population of Fusarium graminearum found in North Dakota is more aggressive and produces a higher level of DON than the prevalent 15ADON population in spring wheat. Phytopathology 100:1007-1014.

35. Qu, B., Li, H. P., Zhang, J. B., Xu, Y. B., Huang, T., Wu, A. B., Carter, J., Nicholson, P., and Liao, Y. C. 2008. Geographical distribution and genetic diversity of the Fusarium graminearum and $F$. asiaticum on wheat spikes throughout China. Plant Pathol. 57:15-24.

36. Schothorst, R. C., and Van Egmond, H. P. 2004. Report from SCOOP task 3.2.10, "Collection of occurrence data of Fusarium toxins in food an assessment of dietary intake by the population of EU member states." Subtask: Trichothecenes. Toxicol. Lett. 153:133-143.

37. Slatkin, M. 1994. Linkage disequilibrium in growing and stable populations. Genetics 137:331-336.

38. Starkey, D. E., Ward, T. J., Aoki, T., Gale, L. R., Kistler, H. C., Geiser, D. M., Suga, H., Toth, B., Varga, J., and O'Donnell, K. 2007. Global molecular surveillance reveals novel Fusarium head blight species and trichothecene toxin diversity. Fungal Genet. Biol. 44:1191-1204

39. Suga, H., Gale, L. R., and Kistler, H. C. 2004. Development of VNTR markers for two Fusarium graminearum clade species. Mol. Ecol. Notes $4: 468-470$

40. Suga, H., Karugia, G. W., Ward, T., Gale, L. R., Tomimura, K., Nakajima, T., Miyasaka, A., Koizumi, S., Kageyama, K., and Hyakumachi, M. 2008. Molecular characterization of the Fusarium graminearum species complex in Japan. Phytopathology 98:159-166.

41. Suzuki, F., Koba, A., and Nakajima, T. 2010. Simultaneous identification of species and trichothecene chemotypes of Fusarium asiaticum and $F$. graminearum sensu stricto by multiplex PCR. J. Gen. Plant Pathol. 76:31-36.

42. Tanaka, T., Hasegawa, A., Yamamoto, S., Lee, U. S., Sugiura, Y., and Ueno, Y. 1988. Worldwide contamination of cereals by the Fusarium mycotoxins nivalenol, deoxynivalenol, and zearalenone. 1. Survey of 19 countries. J. Agric. Food. Chem. 36:979-983.

43. Wang, Y. Z. 1997. Epidemiology and management of wheat scab in China Pages 97-105 in: Fusarium Head Scab: Global Status and Future Prospects. H. J. Dubin, L. Gilchrist, J. Reeves, and A. McNab, eds. CIMMYT, Mexico.

44. Ward, T., J., Bielawski, J. P., Kistler, H. C., Sullivan, E., and O'Donnell, K. 2002. Ancestral polymorphism and adaptive evolution in the trichothecene mycotoxin gene cluster of phytopathogenic Fusarium. Proc. Natl. Acad. Sci. USA 99:9278-9283.

45. Ward, T. J., Clear, R. M., Rooney, A. P., O'Donnell, K., Gaba, D., Patrick, S., Starkey, D. E., Gilbert, J., Geiser, D. M., and Nowicki, T. W. 2008. An adaptive evolutionary shift in Fusarium head blight pathogen populations is driving the rapid spread of more toxigenic Fusarium graminearum in North America. Fungal Genet. Biol. 45:473-484.

46. Windels, C. E. 2000. Economic and social impacts of Fusarium head blight: changing farms and rural communities in the northern Great Plains. Phytopathology 90:17-21

47. Xu, Y. G., and Chen, L. F. 1993. Wheat Scab: Theory and Practice on Control. Jiangsu Science and Technology Publishing House, Nanjing, China.

48. Yang, L., van der Lee, T., Yang, X., Yu, D., and Waalwijk, C. 2008 Fusarium populations on Chinese barley show a dramatic gradient in mycotoxin profiles. Phytopathology 98:719-727.

49. Yeh, F. C., Yang, R. C., Boyle, T., Ye, Z., and Xiyan, J. M. 1997. POPGENE, the user-friendly shareware for population genetic analysis. Molecular Biology and Biotechnology Center, University of Alberta, Canada.

50. Yli-Mattila, T., Gagkaeva, T., Ward, T. J., Aoki, T., Kistler, H. C., and O'Donnell, K. 2009. A novel Asian clade within the Fusarium graminearum species complex includes a newly discovered cereal head blight pathogen from the Russian Far East. Mycologia 101:841-852.

51. Zeller, K. A., Bowden, R. L., and Leslie, J. F. 2003. Diversity of epidemic populations of Gibberella zeae from small quadrats in Kansas and North Dakota. Phytopathology 93, 874-880.

52. Zeller, K. A., Bowden, R. L., and Leslie, J. F. 2004. Population differentiation and recombination in wheat scab populations of Gibberella zeae from the United States. Mol. Ecol. 13:563-571.

53. Zhang, H., Zhang, Z., van der Lee, T., Chen, W. Q., Xu, J., Xu, J. S., Yang, L., Yu, D., Waalwijk, C., and Feng, J. 2010. Population genetic analyses of Fusarium asiaticum populations from barley suggest a recent shift favoring 3ADON producers in southern China. Phytopathology 100:328-336.

54. Zhang, J. B., Li, H. P., Dang, F. J., Qu, B., Xu, Y. B., Zhao, C. S., and Liao, Y. C. 2007. Determination of the trichothecene mycotoxin chemotypes and associated geographical distribution and phylogenetic species of the Fusarium graminearum clade from China. Mycol. Res. 111:967-975.

55. Zhang, Z., Zhang, H., van der Lee, T., Chen, W., Arens, P., Xu, J., Yang, L., Yu, D., Waalwijk, C., and Feng, J. 2010. Geographic substructure of Fusarium asiaticum isolates collected from barley in China. Eur. J. Plan Pathol. 127:239-248.

56. Zhu, H., and Fan, Y. 1989. Wheat scab epidemics and efficacy of testing for resistance in field in northern Fujiang province. Jiangsu Agric. Sci. $1: 75-78$. 